\title{
EXPONENTIAL SUMS OF LERCH'S ZETA FUNCTIONS
}

\author{
KAI WANG
}

ABStraCt. For $x$ not an an integer and $\operatorname{Re}(s)>0$, let

$$
F(x, s)=\sum_{k=1}^{\infty} \frac{e^{2 \pi i k x}}{k^{s}}
$$

be the Lerch's zeta function. In this note, we will show that

$$
\sum_{\gamma=1}^{m-1} e^{-2 \pi i \gamma \alpha / m} F\left(\frac{\gamma}{m}, 1-n\right)=\frac{1}{n}\left(B_{n}-m^{n} B_{n}\left(\frac{\alpha}{m}-\left[\frac{\alpha}{m}\right]\right)\right)
$$

where $\alpha$ is an integer and $\alpha \neq \equiv(\bmod m)$ and $n \geq 1$. For $n=1$, this formula is equivalent to the classical Eisenstein formula

$$
\frac{\alpha}{m}-\left[\frac{\alpha}{m}\right]-\frac{1}{2}=-\frac{1}{2 m} \sum_{\gamma=1}^{m-1} \sin \frac{2 \pi \gamma \alpha}{m} \cot \frac{\pi \gamma}{n} .
$$

1. Introduction. About fifteen years ago, S. Chowla [3] proved the following interesting result:

THEOREM A. Let $p$ be a prime. Then the $\frac{1}{2}(p-1)$ real numbers $\cot \gamma \pi / p, \gamma=$ $1, \ldots, \frac{1}{2}(p-1)$, are linear independent over $Q$, the field of rational numbers.

Recently, we have extended this result to nonprime numbers and the derivatives of all orders of $\cot x[\mathbf{5}]$.

THEOREM B. For an arbitrary positive integer $m$, let $\Lambda=\left\{\gamma \mid 1 \leq \gamma<\frac{1}{2} m\right.$, $(\gamma, m)=1\}$. Then for each $s=0,1, \ldots$, the $\frac{1}{2} \phi(m)$ real numbers

$$
\left\{\left(\frac{d^{s}}{d x^{s}} \cot \frac{(x+\gamma) \pi}{m}\right)_{x=0} \mid \gamma \in \Lambda\right\}
$$

are linear independent over $Q$ where $\phi(m)$ is the Euler function.

On the other hand, there is a classical Eisenstein formula

$$
\frac{\alpha}{m}-\left[\frac{\alpha}{m}\right]-\frac{1}{2}=-\frac{1}{2 m} \sum_{\gamma=1}^{m-1} \sin \frac{2 \pi \gamma \alpha}{m} \cot \frac{\pi \gamma}{m}
$$

Our recent result suggests that it may be of interest to extend the Eisenstein formula to the derivatives of all orders of $\cot x$. Our main result is the following theorem.

Received by the editors June 29, 1984 and, in revised form, November 16, 1984.

1980 Mathematics Subject Classification. Primary 10G05, 10G15; Secondary 10H10. 
THEOREM C. Let $m$ be an arbitrary positive integer. For $s=1$, let $E_{s}=\frac{1}{2}$ and for $s \neq 1$, let $E_{s}=0$. If $s$ is odd and $s \geq 1$, then

$$
\begin{aligned}
\sum_{\alpha=0}^{m-1} \sin \frac{2 \pi \alpha \gamma}{m}\left(\frac{d^{s-1}}{d x^{s-1}} \cot \right. & \left.\left(x+\frac{\alpha \pi}{m}\right)\right)_{x=0} \\
& =\frac{2^{s} i^{s-1}}{s}\left(m^{s} B_{s}\left(\frac{\gamma}{m}-\left[\frac{\gamma}{m}\right]\right)-B_{s}+E_{s}\right) .
\end{aligned}
$$

If $s$ is even and $s \geq 1$, then

$$
\begin{aligned}
\sum_{\alpha=0}^{m-1} \cos \frac{2 \pi \alpha \gamma}{m}\left(\frac{d^{s-1}}{d x^{s-1}} \cot \right. & \left.\left(x+\frac{\alpha \pi}{m}\right)\right)_{x=0} \\
& =\frac{(2 i)^{s}}{s}\left(m^{s} B_{s}\left(\frac{\gamma}{m}-\left[\frac{\gamma}{m}\right]\right)-B_{s},\right.
\end{aligned}
$$

where $\gamma \not \equiv 0(\bmod m), B_{s}$ and $B_{s}(x)$ are Bernoulli numbers and Bernoulli polynomials, respectively.

We refer to $\S 2$ for the definitions of Bernoulli polynomials and Bernoulli numbers.

Theorem $\mathrm{C}$ will be proved as a corollary to Theorem $\mathrm{D}$ in $\S 2$ which involves Lerch's zeta functions.

2. Lerch's zeta functions. Let $\phi(z, s)$ be the power Dirichlet series defined by

$$
\phi(z, s)=\sum_{k=1}^{\infty} \frac{z^{k}}{k^{s}}
$$

which converges for all $s$ if $|z|<1$, for $\operatorname{Re}(s)>0$ if $|z|=1, z \neq 1$, and for $\operatorname{Re}(s)>1$ if $z=1$. It is known that $\phi(z, s)$ can be extended to the whole $s$-plane by means of the contour integral. Let $z=e^{2 \pi i x}$, where $x$ is real, and the periodic function $F(x, s)$ is defined by the equation $F(x, s)=\phi\left(e^{2 \pi i x}, s\right) . F(x, s)$ is called Lerch's zeta function in the literature. For $x$ not an integer and $\operatorname{Re}(s)>0$, we have the series representation

$$
F(x, s)=\sum_{k=1}^{\infty} \frac{e^{2 \pi i k x}}{k^{s}} .
$$

On the other hand, let $B_{n}(x)$ be the $n$th Bernoulli polynomials, defined by the power series

$$
\frac{t e^{x t}}{e^{t}-1}=\sum_{k=0}^{\infty} B_{k}(x) \frac{t^{k}}{k !}
$$

and $B_{n}=B_{n}(0)$ is the $n$th Bernoulli number.

In this note we will prove the following result:

THEOREM D. Let $m>2$ be a fixed positive integer and let $\alpha$ be an integer such that $\alpha \neq \equiv(\bmod m)$. Then, for $n \geq 1$,

$$
\sum_{\gamma=1}^{m-1} e^{-2 \pi i \gamma \alpha / m} F\left(\frac{\gamma}{m}, 1-n\right)=\frac{1}{n}\left(B_{n}-m^{n} B_{n}\left(\frac{\alpha}{m}-\left[\frac{\alpha}{m}\right]\right)\right) .
$$

Our proof involves the Fourier series for the Bernoulli polynomials. This was suggested by a referee of a previous version of this note. 
3. A proof of Theorem D. Let $B_{n}(x)$ be the $n$th Bernoulli polynomial as defined in the Introduction. Then $B_{n}(x-[x])$ is a periodic function of period 1. It is known that the Fourier series of $B_{n}(x-[x])$ are given as follows [4, p. 16]:

$$
B_{2 k-1}(x-[x])=2(-1)^{k}(2 k-1) ! \sum_{h=1}^{\infty} \frac{\sin 2 \pi h x}{(2 \pi h)^{2 k-1}}
$$

and

$$
B_{2 k}(x-[x])=2(-1)^{k-1}(2 k) ! \sum_{h=1}^{\infty} \frac{\cos 2 \pi h x}{(2 \pi h)^{2 k}}
$$

where $B_{1}(x-[x])$ has to be exempted for integral $x$ because the discontinuity of $B_{1}(x-[x])$ at $x$ is equal to 0 . For our purpose, we may combine the above two formulas into one;

$$
B_{n}(x-[x])=-(-i)^{n} n ! \sum_{h=1}^{\infty} \frac{e^{2 \pi i h x}+(-1)^{n} e^{-2 \pi i h x}}{(2 \pi h)^{n}}
$$

for $n>1$.

Recall that $F(x, s)$ satisfies the following functional equation;

$$
F(x, s)=\frac{\Gamma(1-s)}{(2 \pi)^{1-s}}\left(e^{\pi i(1-s) / 2} \xi(1-s, x)+e^{\pi i(s-1) / 2} \xi(1-s, 1-x)\right),
$$

where $\Gamma(s)$ is the gamma function, $\xi(s, x)$ is the Hurwitz zeta function.

Recall that for $0<x \leq 1$ and $\operatorname{Re}(s)>1$,

$$
\xi(s, x)=\sum_{h=0}^{\infty} \frac{1}{(h+x)^{s}} .
$$

Since for $n=1$, Theorem $\mathrm{D}$ is equivalent to Eisenstein's formula, we will only consider the case $n>1$ :

$$
\begin{aligned}
& \sum_{\gamma=1}^{m-1} e^{-2 \pi i \gamma \alpha / m} F\left(\frac{\gamma}{m}, 1-n\right) \\
& =\frac{(n-1) ! i^{n}}{(2 \pi)^{n}}\left(\sum_{\gamma=1}^{m-1} e^{-2 \pi i \gamma \alpha / m} \xi\left(n, \frac{\gamma}{m}\right)+(-1)^{n} \sum_{\gamma=1}^{m-1} e^{-2 \pi i \gamma \alpha / m} \xi\left(n, \frac{m-\gamma}{m}\right)\right) \\
& =\frac{(n-1) ! i^{n}}{(2 \pi)^{n}}\left(\sum_{\gamma=1}^{m-1} e^{-2 \pi \gamma \alpha / m} \xi\left(n, \frac{\gamma}{m}\right)+(-1)^{n} \sum_{\beta=1}^{m-1} e^{2 \pi i \beta \alpha / m} \xi\left(n, \frac{\beta}{m}\right)\right) \\
& =\frac{(n-1) ! i^{n}}{(2 \pi)^{n}}\left(\sum_{\gamma=1}^{m-1} \sum_{k=0}^{\infty} \frac{e^{2 \pi i \gamma \alpha / m}+(-1)^{n} e^{-2 \pi i \gamma \alpha / m}}{(k+\gamma / m)^{n}}\right)
\end{aligned}
$$


(and letting $h=m k+\gamma$ )

$$
\begin{aligned}
& =m^{n}(n-1) ! i^{n} \sum_{h=1, h \neq O(m)}^{\infty} \frac{e^{2 \pi i h \alpha / m}+(-1)^{n} e^{-2 \pi i h \alpha / m}}{(2 \pi h)^{n}} \\
& =m^{n}(n-1) ! i^{n}\left(\sum_{h=1}^{\infty} \frac{e^{2 \pi i h \alpha / m}+(-1)^{n} e^{-2 \pi i h \alpha / m}}{(2 \pi h)^{n}}\right. \\
& \left.-\sum_{k=1}^{\infty} \frac{e^{2 \pi i \alpha k}+(-1)^{n} e^{-2 \pi i \alpha k}}{(2 \pi m k)^{n}}\right) \\
& =m^{n}\left(-\frac{1}{n} B_{n}\left(\frac{\alpha}{m}-\left[\frac{\alpha}{m}\right]\right)+\frac{1}{n m^{n}} B_{n}(\alpha-[\alpha])\right) \\
& =\frac{1}{n}\left(B_{n}-m^{n} B_{n}\left(\frac{\alpha}{m}-\left[\frac{\alpha}{m}\right]\right)\right) .
\end{aligned}
$$

This completes the proof.

4. Examples. The first few values of $F(x,-n)$ are given in [2] as follows:

It follows that

$$
\begin{gathered}
F(x, 0)=-\frac{1}{2}(1-i \cot \pi x), \quad F(x,-1)=-\frac{1}{4}\left(1+\cot ^{2} \pi x\right), \\
F(x,-2)=-\frac{i}{8}\left(2 \cot \pi x+2 \cot ^{3} \pi x\right) .
\end{gathered}
$$

$$
\begin{aligned}
& \sum_{\alpha=1}^{m-1} \cos \frac{2 \pi \alpha \gamma}{m} \cot ^{2} \frac{\alpha \pi}{m}=\frac{2}{3}+2 m^{2} B_{2}\left(\frac{\gamma}{m}-\left[\frac{\gamma}{m}\right]\right) \\
& \sum_{\alpha=1}^{m-1} \sin \frac{2 \pi \alpha \gamma}{m} \cot ^{3} \frac{\alpha \pi}{m}=-2 m B_{1}\left(\frac{\gamma}{m}-\left[\frac{\gamma}{m}\right]\right)+\frac{4 m^{3}}{3} B_{3}\left(\frac{\gamma}{m}-\left[\frac{\gamma}{m}\right]\right) .
\end{aligned}
$$

5. A proof of Theorem C. Now Theorem C follows easily from Theorem $D$ because

$$
F(x, 0)=\frac{1}{2}(1-i \cot \pi x) .
$$

6. Comments. (1) Theorem $\mathrm{C}$ is equivalent to the following:

THEOREM E. Let $m>2$ be a fixed positive integer and let $\alpha$ be an integer such that $\alpha \not \equiv 0(\bmod m)$. Then,

$$
\begin{aligned}
& \frac{1}{2 i} \sum_{\gamma=1}^{m-1} e^{-2 \pi i \alpha \gamma / m} \cot \left(x+\frac{\gamma \pi}{m}\right) \\
& \quad=\frac{1}{2}-\frac{e^{2 x i}}{e^{2 x i}-1}+\frac{m e^{2 m x i}(\gamma / m-[\gamma / m])}{e^{2 m x i}-1} .
\end{aligned}
$$

This formula was originally proved by different arguments without involving Lerch's zeta function. The computation is considerably longer.

(2) Theorem $\mathrm{D}$ can also be used to derive the formula for Dirichlet $L$-function using the following representation of Dirichlet $L$-function for primitive $\bmod m$ Dirichlet character $\chi[\mathbf{2}]$ :

$$
\tau(\bar{\chi}) L(s, \chi)=\sum_{\alpha=1}^{m-1} \bar{\chi}(\alpha) F\left(\frac{\alpha}{m}, s\right),
$$

where $\tau(\bar{\chi})$ is the Gauss sum. 


\section{REFERENCES}

1. T. M. Apostol, Introduction to analytic number theory, Springer-Verlag, Berlin and New York, 1976.

2. _ Dirichlet L-functions and character power sums, J. Number Theory 2 (1970), 223-234.

3. S. Chowla, The nonexistence of nontrivial linear relations between roots of a certain irreducible equation, J. Number Theory 2 (1970), 120-123.

4. H. Rademacher, Topics in analytic number theory, Springer-Verlag, Berlin and New York, 1973.

5. K. Wang, On a theorem of $S$. Chowla, J. Number Theory 15 (1982), 1-4.

Department of Mathematics, Wayne State University, Detroit, Michigan 48202 